\title{
Poor sporting abilities after tibial plateau fractures involving the posterior column: how can we do better?
}

\author{
Liselore Quintens ${ }^{1}$ - Juriaan Van den Berg ${ }^{1} \cdot$ Maike Reul $^{2} \cdot$ Esther Van Lieshout $^{3} \cdot$ Stefaan Nijs $^{1} \cdot$ Michiel Verhofstad $^{3}$. \\ Harm Hoekstra ${ }^{1}$ (i)
}

Received: 8 March 2019 / Accepted: 26 August 2019

(c) Springer-Verlag GmbH Germany, part of Springer Nature 2019

\begin{abstract}
Purpose Tibial plateau fractures with the involvement of the posterior column are an important prognostic factor towards poor functional outcome. We aimed to assess the sporting abilities postoperatively with special emphasis on the type of sports and sport-specific movements, as well as time needed to resume sports, restricting factors in sports engagement, and patient satisfaction. We aimed to provide prognostic information on return to sports.

Methods Demographic, clinical and radiological variables were retrospectively collected from 82 multicentric patients between 2014 and 2016. Prospectively, sporting abilities before and after surgery were determined using questionnaires at a mean follow-up of 33 months postoperatively.

Results Involvement in sports significantly decreased, with only $68.4 \%$ of patients resuming sports $(p<0.001)$. The mean time needed to partially or fully resume sports was 6-9 and 9-12 months, respectively. The ability to resume at the pre-injury level of effort and performance was $22 \%$ and $12 \%$, respectively. Restricting factors were pain (66\%), fear of re-injury (37\%), limited range of motion (26\%), and instability (21\%). The majority (59\%) of patients were unsatisfied with their physical abilities. Significantly worse outcomes were observed in patients playing high-impact sports, experiencing knee pain during physical activity, suffering from extension/valgus or flexion/varus trauma.

Conclusions Tibial plateau fractures with the involvement of the posterior column significantly hamper the patients' sporting abilities, leaving the majority of patients unsatisfied. Preoperative counseling about prognosis, setting realistic expectations, optimizing rehabilitation and pain management postoperatively, and advising low-impact sports might improve engagement in physical activities and emotional impact on patients.
\end{abstract}

Level of evidence 3 .

Keywords Knee $\cdot$ Trauma $\cdot$ Fracture $\cdot$ Posterior column $\cdot$ Physical activity $\cdot$ Sports

Harm Hoekstra

harm.hoekstra@uzleuven.be

1 Department Trauma Surgery, University Hospital Leuven, KU Leuven, University of Leuven, Herestraat 49, 3000 Leuven, Belgium

2 Department of Orthopedics and Traumatology, VU Brussel, Free University of Brussels, Brussels, Belgium

3 Trauma Research Unit Department of Surgery, Erasmus MC, University Medical Center Rotterdam, Rotterdam, The Netherlands

\section{Introduction}

Tibial plateau fractures account for $1 \%$ of all fractures and are typically sustained by high-energy traumas [1]. The consequences of tibial plateau fractures can be far reaching, especially for those who suffered from a more severe type, requiring extensive surgery [2-4]. Tibial plateau fractures with the involvement of the posterior column have been identified as an important prognostic factor towards poor outcome [5].

Assessing functional outcome after a tibial plateau fracture, patients reported significantly lower scores on the sports and recreation subscales of the Knee injury and Osteoarthritis Outcome Score (KOOS) [3-5]. Since the majority of patients sustaining these injuries are relatively young and 
active, this can have devastating consequences for their leisure or competitive involvement in sports. Adequate physical activity is important among all ages, and its health benefits for both physical and emotional wellbeing have been well established [6-9]. While clinical and radiological outcomes of tibial plateau fractures are elaborately studied, there has been very little appreciation for the impact of these injuries on the patient's physical lifestyle, in particular their ability to participate in sports. In studies using validated functional outcome scores, return to sports is often only a secondary outcome measure. Therefore, detailed description of physical limitations is lacking.

The handful of studies that do elaborate on the sequelae after tibial plateau fractures with regard to return to sports seem to agree that the majority of patients are not able to return to their previous level of activity. For patients playing competitive sports, this injury can be a career ender [10-12]. The complexity of the fracture and the age at the time of the injury seem to be an important prognostic factor for the final sporting abilities [11, 12]. A recent study demonstrated a weak correlation between the physical abilities after these injuries and the range of knee motion and postoperative infection rates [11]. However, comprehensive information on return rates, detailing the type of sports and sport-specific movements that are mostly affected by the injury, is not available. Moreover, insight into the time it takes to return to both training and full-level sports, and the emotional impact on the patients is lacking. Finally, to our knowledge, no research has been performed into the causes of these physical limitations, which is crucial information for setting up early interventions and an adequate rehabilitation program.

Therefore, we aimed to provide comprehensive information on these four aspects, as well as provide prognostic information on return to sports after these fractures.

\section{Methods}

\section{Patients}

This multicenter study has been conducted throughout three level 1 trauma centers. After identification of all consecutive patients operatively treated for a tibial plateau fracture between 2014 and 2016, only those with (1) involvement of the posterior column according to the revised three-column concept (rTCC) classification [13] and (2) a closed epiphysis were included. Exclusion criteria were non-articular fractures, bilateral fractures, patients with accompanying injuries in the same extremity, pathologic fractures, deceased patients, and patients missing CT scan preoperatively. According to these criteria, a total of 82 patients were available. Informed consent was obtained from all individual participants included in the study, comprising a total of 51 patients. The study protocol was approved by all local medical research ethics committees (Ethische Commissie Onderzoek UZ/KU Leuven, Commissie Medische Ethiek and Medische Ethische Toetsings Commissie Erasmus MC) and the rights of the subjects were protected.

\section{Demographics and clinical characteristics}

A total of 26 demographic, clinical and radiological variables were retrieved from the hospitals' electronic medical file databases. The demographic characteristics recorded were age, gender, ASA score, BMI, smoking status, medication status, diabetes status and other cardiovascular risk factors. Trauma and treatment variables that were recorded included trauma mechanism, open vs. closed fractures, time to surgery, external fixation use (either as definite treatment or in staged surgery) and period of non-full-weight bearing. All fractures were classified using the rTCC approach, to depict posterior column fractures and track the total number of fractured columns [13]. Recorded complications were categorized as fracture-related infection, non-union and reintervention. Postoperatively, coronal alignment, sagittal alignment, condylar width and articular congruence were measured.

\section{Outcome measures}

Prospectively, sporting abilities before and after surgery were determined using questionnaires. The questionnaires included the standardized and validated versions of the KOOS questionnaire for the Dutch [14] or French [15] language, as well as 23 sport-related questions. These sportrelated questions assessed sporting frequency and level in the year before the injury and at the time of the survey. In addition, questions were included regarding the specific sports played, the ability to perform specific movements according to the Activity Rating Scale (ARS) for disorders of the knee [16-18], the perceived limitations in effort and performance, the time needed to resume physical activities, the perceived reasons for limitations, and the satisfaction with ones sporting abilities. Concerning the ARS, 'running' is interpreted as running while playing a sport or jogging, 'cutting' is interpreted as changing directions while running, 'decelerating' is interpreted as coming to a quick stop while running, and 'pivoting' is interpreted as turning your body with your foot planted while playing a sport. To compare our KOOS scores with an average adult population, the population-based cohort of Paradowski et al. was used as a reference [19]. 


\section{Statistical analysis}

Statistical analysis was performed using IBM SPSS 25.0. Normal distribution was tested in continuous data using a Shapiro-Wilk test. Continuous data are shown as mean with SD (if parametric) or as median with $P_{25}-P_{75}$ (if nonparametric). Normally distributed data were compared using $t$ tests, and for nonparametric variables, a Mann-Whitney $U$ test was used. Chi-square statistics were used to compare nominal variables. Nominal variables that did not comply with the assumptions for a chi-square test were compared using a Fisher's exact test. Predictive analysis was performed using multiple logistic regression. A significance level of $<0.05$ was accepted for all tests.

\section{Results}

\section{Descriptives}

A total of 51 patients were included in this study. The average age of the cohort was $51( \pm 14)$ years, comprising a total of 17 men and 34 women, with a mean follow-up time of 33 months and a minimal follow-up of 14 months. All demographic, clinical and radiographic variables are displayed in Table 1. These were compared with the variables of the patients that did not return a written informed consent. Only the BMI differed significantly between those that did and those that did not return a written informed consent, $26.5 \pm 4.8$ and $24.6 \pm 3.9(p=0.036)$, respectively. The KOOS scores for the study population are displayed in Fig. 1, and compared with reference values from an average adult population [19], and values from an average population of tibial plateau fractures treated with ORIF [3].

\section{Sports and recreational activities}

A total of 29 patients (57\%) were engaged in sports throughout the year before the injury, either on recreational, competitive or professional level. Thirty-one percent of patients were active 2-3 times a week, 16\% 4-5 times a week and $10 \%$ more than 5 times a week. Only three patients played on a competitive or professional level before the injury. According to the ARS, several specific movements stressing the knee were further analyzed. During their activities, $44 \%$ of the physically active patients ran during their physical activities, $44 \%$ performed cutting movements and quick directional changes, $28 \%$ performed quick decelerations and $50 \%$ performed rotational movements pivoting on their knees.

At the end of the follow-up, only $39 \%$ of the patients were engaged in sports, indicating that only two out of three patients playing sports before the injury are still able to do so after, illustrating a significant decrease $(p<0.001)$.
Table 1 Demographic and clinical characteristics

\begin{tabular}{|c|c|}
\hline & $\begin{array}{l}\text { Mean }( \pm \mathrm{SD}), \\
\text { median }\left(P_{25}-P_{75}\right) \text { or } \\
n(\%)\end{array}$ \\
\hline Age (years) & $51( \pm 13.7)$ \\
\hline \multicolumn{2}{|l|}{ Gender } \\
\hline Female & $34(67 \%)$ \\
\hline \multicolumn{2}{|l|}{ ASA } \\
\hline 1 & $19(37 \%)$ \\
\hline 2 & $29(57 \%)$ \\
\hline 3 & $3(6 \%)$ \\
\hline $\operatorname{BMI}\left(\mathrm{kg} / \mathrm{m}^{2}\right)$ & $26.2(23.5-28.2)$ \\
\hline Smoker & $11(22 \%)$ \\
\hline Taking medication & $13(25 \%)$ \\
\hline Diabetes & $6(12 \%)$ \\
\hline Other CVRF & $12(24 \%)$ \\
\hline \multicolumn{2}{|l|}{ Trauma mechanism } \\
\hline Extension/valgus & $18(35 \%)$ \\
\hline Extension/varus & $6(12 \%)$ \\
\hline Flexion/valgus & $15(29 \%)$ \\
\hline Flexion/varus & $12(24 \%)$ \\
\hline \multicolumn{2}{|l|}{ Open fracture } \\
\hline Yes & $5(10 \%)$ \\
\hline \multicolumn{2}{|l|}{ rTCC } \\
\hline 1 column & $3(6 \%)$ \\
\hline 2 columns & $21(41 \%)$ \\
\hline 3 columns & $27(53 \%)$ \\
\hline Lateral column & $46(90 \%)$ \\
\hline Medial column & $29(57 \%)$ \\
\hline \multicolumn{2}{|l|}{ Treatment parameters } \\
\hline Delayed staged surgery & $9(18 \%)$ \\
\hline Time to surgery (days) & $4(2-8)$ \\
\hline Period of non-full-weight bearing (days) & $12(8-12)$ \\
\hline \multicolumn{2}{|l|}{ Complications } \\
\hline Any complication & $19(37 \%)$ \\
\hline Infection & $10(20 \%)$ \\
\hline Nonunion & $2(4 \%)$ \\
\hline Other complication & $7(13 \%)$ \\
\hline Re-intervention & $24(47 \%)$ \\
\hline Implant removal & $18(35 \%)$ \\
\hline Revision & $9(18 \%)$ \\
\hline \multicolumn{2}{|l|}{ Radiology } \\
\hline Radiologic failure & $33(65 \%)$ \\
\hline Coronal malalignment (MPTA $87 \pm 5$ ) & $7(14 \%)$ \\
\hline Sagittal malalignment (PPTA 9 \pm 5 ) & $7(14 \%)$ \\
\hline Condylar width $(<0 \mathrm{~mm},>5 \mathrm{~mm})$ & $7(14 \%)$ \\
\hline Articular incongruence (> $2 \mathrm{~mm})$ & $23(45 \%)$ \\
\hline
\end{tabular}

Other complications are deep venous thrombosis, neuralgia, soft tissue swelling with blisters, and dehiscence

ASA American Society of Anesthesiologists, BMI body mass index, $C V R F$ cardiovascular risk factors, $r T C C$ revised three-column classification, MPTA medial proximal tibia angle, PPTA posterior proximal tibial angle 
Fig. 1 The KOOS subscales, comparing a reference population [19], and an average population of tibial plateau fractures treated with ORIF (14), with our cohort of 51 patients who suffered an operatively treated tibial plateau fracture involving the posterior column. KOOS Knee injury and Osteoarthritis Outcome Score, $A D L$ activities of daily living, Sport/Rec function in sport and recreation, $Q o L$ knee-related quality of life

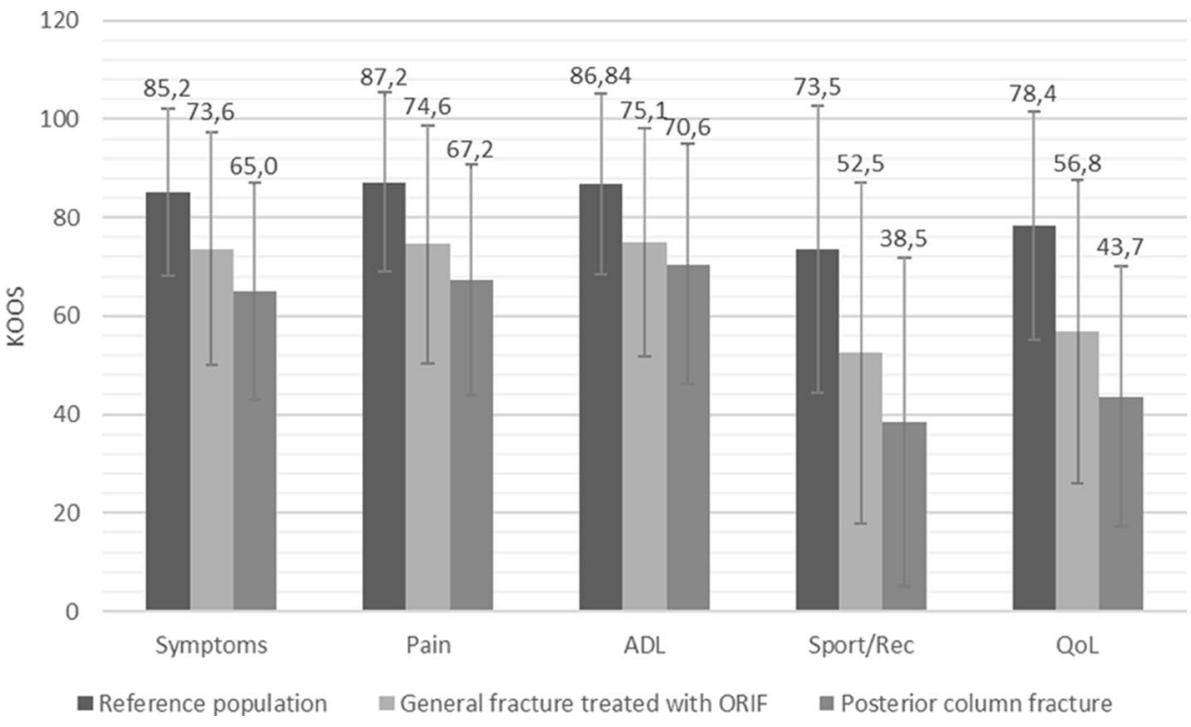

As much as 55\% of all patients reduced their frequency of physical activity. The mean time needed to partially resume pre-injury activities was 6-9 months. Sixteen percent of the responding patients were not able to resume their activities. For those able to fully resume, the mean time needed to do so was 9-12 months. However, as much as $41 \%$ did not fully return to their pre-injury sports.

The decrease in knee-stressing movements performed during physical activity was more pronounced than the decrease in patients engaged in sports. Only $16 \%$ still ran during their physical activities after the injury, $19 \%$ of the patients still performed cutting movements and quick directional changes, $13 \%$ still performed quick decelerations and $25 \%$ still performed rotational movements pivoting on their knee.

Self-reported ability to resume a sport at the pre-injury level of effort and performance was poor. $78 \%$ of patients reported not being able to fully engage and as much as $88 \%$ reported they were not able to reach the same level of performance.

\section{Importance of type of sport}

Forty-seven percent of the patients engaged in physical activity before their injury played at least one high-impact sport (tennis, squash, horseback riding, snowboarding, skiing, waterskiing, running or dancing). Thirty-four percent of the patients engaged in physical activity were only engaged in low-impact sports (cycling, swimming or hiking). Nineteen percent did not disclose their type of physical activity.

Patients who played only low-impact sports were more likely to return to it than those engaged in high-impact sports [ 9 out of $11(82.8 \%)$ versus 6 out of $15(40 \%)$; $p=0.043$ ] (Fig. 2). The odds of being able to return to
Fig. 2 Importance of the type of sports, comparison between high- and low-impact sports with a the percentage of patients able to return to sports, $\mathbf{b}$ the percentage of patients able to resume a sport at the pre-injury level of effort, $\mathbf{c}$ the percentage of patients not experiencing pain in the knee during physical activity, and $\mathbf{d}$ the percentage of patients satisfied with the physical abilities

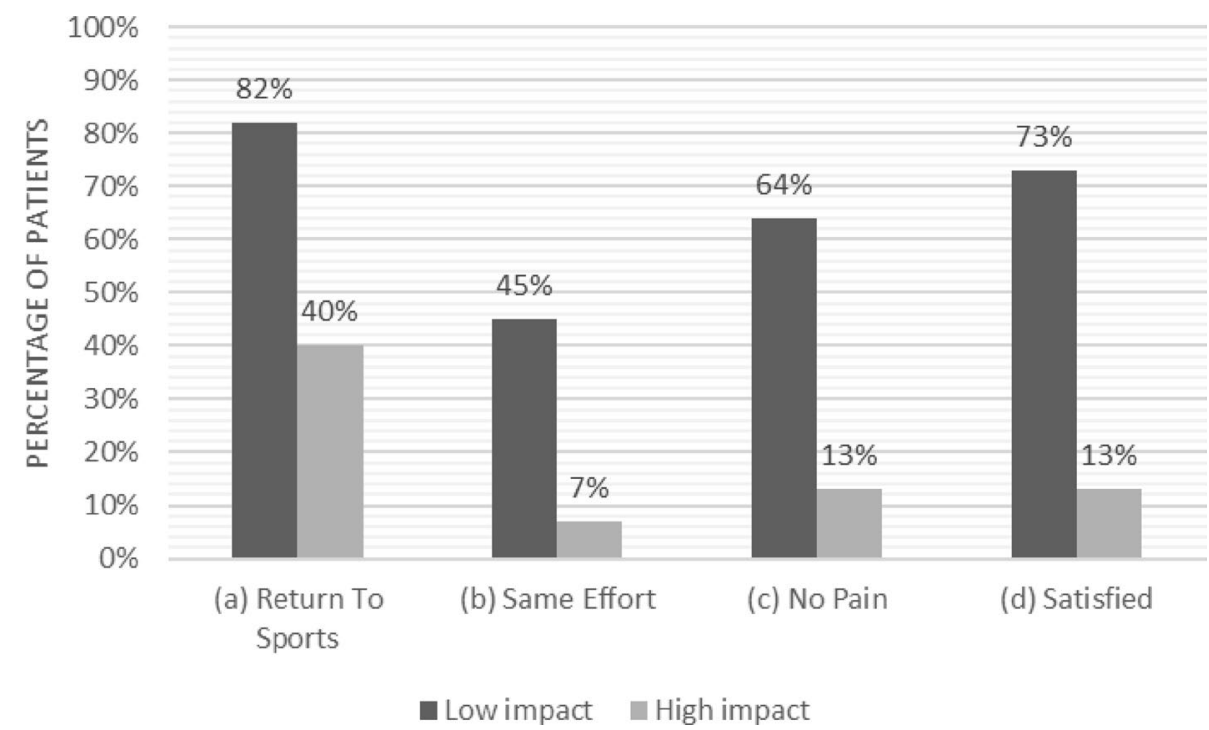


sports were 6.8 times higher. Of all patients, only two increased their frequency of activities. Both concerned elderly people, aged 60 and 75 years. Nevertheless, they did not disclose their type of physical activity and considering their age, it is most likely they participated in lower impact sports. Three patients who were not engaged in physical activity before their injury, stated to have picked up sports afterwards. All three picked up only low-impact sports.

Being engaged in low-impact sports was associated with a sooner partial, as well as full resumption of preinjury sports, compared with high-impact sports. At 6-9 months and 9-12 months after the injury, the mean time needed to partially and fully, respectively, resume sport activities, patients playing low-impact sports succeeded 7.0 times and 7.2, respectively, times more often to resume their pre-injury sports than those patients playing high-impact sports [6 out of $11(54.5 \%)$ versus 3 out of $15(20 \%) ; p=0.031$ resp. 7 out of $11(63.6 \%)$ versus 3 out of $15(20 \%) ; p=0.043$, respectively].

Finally, a better outcome for self-reported ability to resume a sport at the pre-injury level of effort was associated with lower impact sports as well [5 out of $11(45.5 \%)$ versus 1 out of 15 (6.7\%); $p=0.040$ ] (Fig. 2). Although a low percentage of patients were able to engage with the same level of effort, patients playing low-impact sports were 11.7 times more likely to be able to do so than patients playing high-impact sports. Self-reported ability to resume a sport at the pre-injury level of performance is not associated with the impact of the sports played $[2$ out of $11(18.2 \%)$ versus 1 out of $15(6.7 \%) ; p=0.382$ ]. Both patients engaged in low- and high-impact sports were very likely to not be able to do so.

\section{Perceived causes for limitations in sport engagement}

All patients, regardless of their ability to return to sports, were questioned about knee- and non-knee-related factors they felt were restricting them from fully engaging in physical activities. The most important knee-related factors were pain in the knee, fear of either hurting or re-injuring the knee, limitations in the range of motion and instability of the knee (Fig. 3). 16\% of the patients experienced non-kneerelated factors. Patients suffering pain during sports were 11 times more likely to not return to sports than those who did not experience pain during physical activity [ 11 out of 21 (52.4\%) versus 1 out of $11(9.1 \%) ; p=0.035]$. No significant association between the other factors, the amount of factors, and the ability to return to sports was found.

Patients engaged in higher impact sports were 11.4 times more likely to suffer from pain-related knee problems during physical activity than patients only engaged in low-impact sports [13 out of $15(86.7 \%)$ versus 4 out of 11 (36.4\%); $p=0.014$ ] (Fig. 2). The occurrence of the other knee- and non-knee-related limitations was not significantly associated with the impact of the sports played.

\section{Satisfaction}

Thirty-one percent of the patients were very disappointed, $28 \%$ were slightly disappointed, $19 \%$ were satisfied, and $22 \%$ were very satisfied with the outcome. Patients were more likely to be unsatisfied with their physical abilities when not being able to resume sports [ 11 out of 12 (91.7\%) compared to 8 out of $20(40 \%) ; p=0.014]$ or play at pre-injury level of effort [19 out of $25(76.0 \%)$ compared to 0 out of $7 ; p<0.001]$ or performance [19 out of
Fig. 3 Restricting factors in sports engagement. ROM range of motion

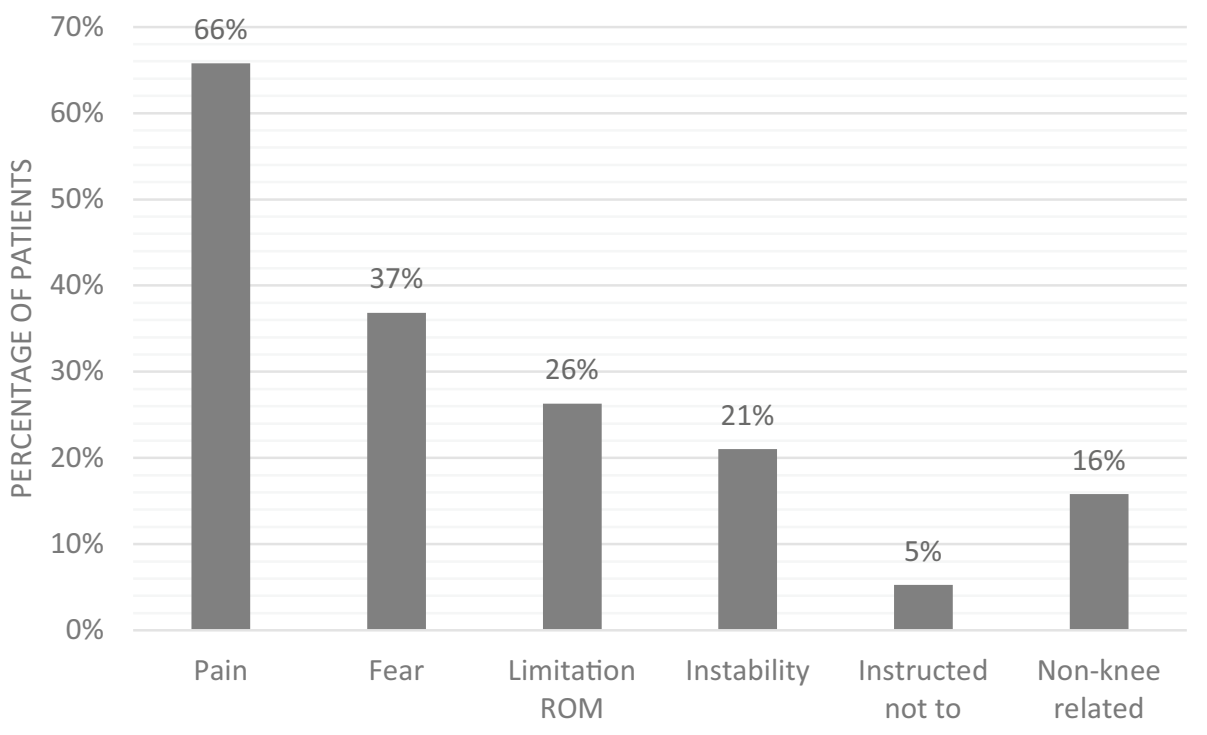


28 (67.9\%) compared to 0 out of $4 ; p<0.001]$. Experiencing pain in the knee during physical activity, or experiencing two or more of above-mentioned causes for limitations, were also associated with a lower satisfaction rate [19 out of $21(90.5 \%)$ compared to 0 out of $11 . p<0.001$ resp. 15 out of 17 (88.2\%) compared to 4 out of 14 (28.6\%); $p=0.002]$. Playing a high-impact sport was again associated with worse outcomes, as the odds of being unsatisfied for patients playing a high-impact sport were 17.2 times higher compared to patients playing a low-impact sport [13 out of $15(86.7 \%)$ compared to 3 out of 11 (27.3\%); $p=0.005]$ (Fig. 2).

\section{Factors predicting sporting abilities}

Bivariate analysis on ability to return to sports and the reduction in frequency of physical activity after the injury was performed in regard to all demographic, clinical and radiological parameters. All results are presented in Table 2. No predicting factors for return to sports could be established since all bivariate significant results lost their significant $p$ value when considered in a multivariable model. For frequency of physical activity, trauma mechanism proved to be a predicting factor. A flexion/valgus trauma was associated with better outcomes than an extension/valgus trauma or a flexion/varus trauma [9 out of $10(90 \%)$ versus 3 out of

\begin{tabular}{|c|c|c|}
\hline & Return to sports & $\begin{array}{l}\text { Reduction in fre- } \\
\text { quency of physical } \\
\text { activity }\end{array}$ \\
\hline \multicolumn{3}{|l|}{ Demographics } \\
\hline Age (years) & 0.087 & 0.200 \\
\hline Gender & 0.999 & 0.999 \\
\hline ASA score & 0.518 & 0.427 \\
\hline BMI & 0.392 & 0.291 \\
\hline Smoking & 0.999 & 0.484 \\
\hline Medication & 0.372 & 0.394 \\
\hline Diabetes & 0.516 & 0.484 \\
\hline Other CVRF & 0.626 & 0.999 \\
\hline \multicolumn{3}{|l|}{ Trauma and fracture } \\
\hline Trauma mechanism & $0.010^{*}$ & $<0.001 *$ \\
\hline Open fracture & 0.620 & 0.999 \\
\hline Number of columns according to rTCC & 0.458 & 0.225 \\
\hline Involvement lateral column & 0.516 & 0.484 \\
\hline Involvement medial column & 0.713 & 0.473 \\
\hline \multicolumn{3}{|l|}{ Treatment parameters } \\
\hline Delayed staged surgery & 0.620 & 0.101 \\
\hline Time to surgery (days) & 0.243 & 0.223 \\
\hline Period of non-full-weight bearing (days) & 0.390 & 0.183 \\
\hline Any complication & 0.713 & 0.999 \\
\hline Infection & 0.999 & 0.999 \\
\hline Nonunion & 0.516 & 0.484 \\
\hline Re-intervention rate & 0.726 & 0.479 \\
\hline Implant removal & 0.288 & 0.716 \\
\hline Revision & 0.683 & 0.394 \\
\hline \multicolumn{3}{|l|}{ Radiological outcome } \\
\hline Radiological failure & 0.724 & 0.716 \\
\hline Coronal malalignment (MPTA $87 \pm 5$ ) & 0.999 & 0.999 \\
\hline Sagittal malalignment (PPTA 9 \pm 5 ) & 0.629 & 0.333 \\
\hline Condylar width $(<0 \mathrm{~mm},>5 \mathrm{~mm})$ & 0.338 & 0.999 \\
\hline Articular incongruence (>2 mm) & 0.726 & 0.479 \\
\hline
\end{tabular}

Significant data are indicated with an *

ASA American Society of Anesthesiologists, BMI body mass index, CVRF cardiovascular risk factors, rTCC revised three-column classification, MPTA medial proximal tibia angle, PPTA posterior proximal tibial angle 
$10(30 \%) ; p=0.003$ resp. 9 out of $10(90 \%)$ versus 5 out of $9(55.6 \%) ; p=0.023]$.

\section{Discussion}

While clinical and radiological outcomes of tibial plateau fractures have elaborately been studied, there has been very little appreciation for the impact of tibial plateau fractures on the patient's physical abilities. Therefore, the purpose of this study was to provide a more comprehensive description of the sequelae regarding sporting abilities after tibial plateau fractures involving the posterior column along with providing prognostic information on return to sports.

Our results indicate that tibial plateau fractures with involvement of the posterior column indeed significantly hamper the patients' sporting abilities, leaving the majority of patients unsatisfied. Despite moderate results with regard to the other KOOS subscales, patients scored indeed significantly lower on the sport and recreation subscale. Patients were likely to reduce their involvement in physical activity, scored significantly lower on the ARS, and a vast majority was not able to resume their sports at pre-injury level of effort nor performance. Worse outcome scores were observed in patients playing high-impact sports and in patients experiencing pain in the knee during physical activity. Trauma mechanisms that proved to be associated with a reduction in frequency of physical activity were extension/ valgus trauma and flexion/varus trauma.

Previously, Kraus et al. and Loibl et al. [10, 12] reported $82 \%$ and $88 \%$ return to sport rates in a general cohort of tibial plateau fractures. We observed a return rate of only $68 \%$ after a mean follow-up of 33 months. Disparities in fracture types and treatment techniques are important to consider here since more severe fractures are associated with poor outcome $[10,12]$. We included tibial plateau fractures with the involvement of the posterior column since posterior column fractures (and associated malalignment) have been identified as an important prognostic factor towards the poor outcome [5]. Kugelman et al. [11] reported even a lower return to sports rate of $52.4 \%$. However, the mean follow-up in their study was only 15 months. Kraus et al. [10] demonstrated a significant increase in return to sports at a followup of 52.8 months after surgery, compared to the physical activity 1 year postoperatively $(p<0.001)$. Furthermore, in our study, to our knowledge the first to report on the time needed to partially and fully return to sports, the mean time needed was 6-9 months and 9-12 months, respectively. This affirms the extensive rehabilitation needed for these fractures, with resumption of physical activity often outdating the standard follow-up for these injuries. Therefore, return to sports measured by Kugelman et al., and to a lesser extent in this study, is likely to further increase over time. Though these recent studies all demonstrated an overall good clinical result and rate of return to sports, patients were, in line with our results, very likely to give up ambitious sports activities [10-12]. In our cohort, $78 \%$ of patients reported they were not able to resume sport at the pre-injury level of effort and as much as $88 \%$ reported they were not able to reach the same level of performance.

Kraus et al. [10] were the first to point out a shift away from high towards lower impact activities. However, further analysis was lacking. We observed that patients playing high-impact sports proved a high-risk group, being almost 7 times more likely to not return to sports, having a longer rehabilitation period, and being 11.7 times less likely to play at the pre-injury level of effort, 11.4 times more likely to suffer from pain-related knee problems during physical activity and 17.2 times more likely to be unsatisfied with their surgical results. Counseling these patients about their post-injury prognosis, providing early interventions focusing on pain management and advising lower impact sports might, therefore, be beneficial for the functional outcome and the emotional impact on the patients. Moreover, it is known that the knee is a heavily stressed joint. Kuster et al. [20] described the peak loads in the knee joint being 1.2 times the body weight during cycling and 3-4 times the body weight during walking. Higher impact sports on the contrary showed much higher peak loads of 5-6 times the body weight during squatting, up to 10 times during downhill skiing and up to as much as 14 times during jogging and running [20]. Considering the degenerative articular changes and the fact that higher stresses might cause more pain, minimizing the peak loads on the knee during physical activity can be considered beneficial, and an extra argument to counsel the patients towards lower impact sports.

Crucial for setting up early interventions is getting insight into why people are not able to resume their physical activities. To our knowledge, we are the first to investigate the perceived causes for these limitations. A range of reasons were pointed out, such as pain in the knee, fear of re-injury, limitations in range of motion, and instability of the knee. All of these are potentially adjustable, therefore, important to focus on during rehabilitation. However, only pain in the knee during physical activities was significantly associated with the inability to return to sports. Patients experiencing pain during sports were as much as 11 times more likely to be unable to return to sports compared to patients without any pain, denoting adequate pain management an important issue to be addressed by the physician.

A wide variety of demographic, clinical and radiological variables were investigated for their predicting ability on return to sport. Loibl et al. [12], as well as Kugelman et al. [11], found that a higher age at the time of the injury was associated with a lower return to sports, arguing that an advancing age might be related to a reduced 
physiological reserve and healing potential. However, neither our study nor Kraus et al.'s [10] found a significant difference between age groups. Furthermore, we found no other demographic variables that had a predicting ability on whether or not the patient was likely to return to sports. Considering the clinical variables, there is some evidence for a better outcome with the use of an external fixator combined with percutaneous large-fragment lag screws as definite treatment, compared to open reduction and internal fixation [21]. Return to sports was higher at 6 months $(p=0.031)$ and 12 months $(p=0.024)$ after the injury when external fixation was used to treat the fracture [21]. The authors argue that less soft tissue damage might be responsible for the better outcome. This difference, however, disappeared at 24 months after the fracture ( $p=0.128)$, and other long-term follow-up studies could not conclude a significant difference [22]. This is in line with our results. Notwithstanding the fact that soft tissue injury was not scored in this retrospective study, delayedstaged surgery using a temporary external fixation device was performed in nine cases. Moreover, delayed-staged surgery was not associated with less return to sports. The only clinical variable associated with reduction in frequency of physical activity was the trauma mechanism. Extension/valgus trauma and flexion/varus trauma were associated with worse outcome compared to a flexion/valgus trauma. Regarding the radiological variables finally, the importance of preventing postoperative knee malalignment is widely accepted [23-25]. However, we found no significant influence on the ability to return to sports.

The present study has a number of limitations. A first limitation is the retrospective design. As patients were asked about their physical activities in the year before the injury, raising the possibility of a recall bias since this, on average, dates back several years. Also, because the patients were several years older at the time of the followup, there might have been a decrease in engagement in physical activity independent of the injury, as patients tend to become less active with increasing age. Furthermore, $38 \%$ of patients did not return the questionnaire, which might cause a selection bias. However, apart from BMI, there were no other statistically significant differences in any of the other demographic, clinical or radiographic variables between responders and non-responders. Although this type of fractures was only treated by experienced orthopedic trauma surgeons in three level 1 trauma centers, there is a possible treatment bias involved. Finally, a subgroup analysis for the level of sports participation could not be performed, as only three patients played on a competitive or professional level. The strengths of the present study are the unique cohort, the detailed description of physical activities, the new prognostic insights and a long follow-up period.

\section{Conclusion}

In conclusion, tibial plateau fractures with the involvement of the posterior column significantly hamper the patients' sporting abilities, leaving the majority of patients unsatisfied and unable to play at the pre-injury level of effort and performance. Patients, especially high-risk groups such as patients playing high-impact sports, should be counseled appropriately about the prognosis, setting realistic expectations. Moreover, early interventions and adequate rehabilitation programs focusing on pain management should be provided. Advising lower impact sports might also be beneficial for improving the engagement in physical activities and the emotional impact on the patients.

Funding There is no funding source.

\section{Compliance with ethical standards}

Conflict of interest The authors declare that they have no conflict of interest.

Ethical approval This study was completed in compliance with national legislation and the guidelines of the Ethische Commissie Onderzoek UZ/KU Leuven, Commissie Medische Ethiek and Medische Ethische Toetsings Commissie Erasmus MC. The rights of the subjects were protected.

Informed consent Informed consent was obtained from all individual participants included in the study.

Statement of human rights All procedures performed in studies involving human participants were in accordance with the ethical standards of the institutional and/or national research committee and with the 1964 Helsinki declaration and its later amendments or comparable ethical standards.

\section{References}

1. Court-Brown CM, Caesar B. Epidemiology of adult fractures: a review. Injury. 2006;37(8):691-7.

2. Manidakis N, Dosani A, Dimitriou R, Stengel D, Matthews S, Giannoudis P. Tibial plateau fractures: functional outcome and incidence of osteoarthritis in 125 cases. Int Orthop. 2010;34(4):565-70.

3. Timmers TK, van der Ven DJ, de Vries LS, van Olden GD. Functional outcome after tibial plateau fracture osteosynthesis: a mean follow-up of 6 years. Knee. 2014;21(6):1210-5.

4. van Dreumel RL, van Wunnik BP, Janssen L, Simons PC, Janzing HM. Mid- to long-term functional outcome after open reduction and internal fixation of tibial plateau fractures. Injury. 2015;46(8):1608-12.

5. van den Berg J, Reul M, Nunes Cardozo M, Starovoyt A, Geusens E, Nijs S, et al. Functional outcome of intra-articular tibial plateau fractures: the impact of posterior column fractures. Int Orthop. 2017;41(9):1865-73.

6. Garber CE, Blissmer B, Deschenes MR, Franklin BA, Lamonte MJ, Lee IM, et al. American College of Sports Medicine position 
stand. Quantity and quality of exercise for developing and maintaining cardiorespiratory, musculoskeletal, and neuromotor fitness in apparently healthy adults: guidance for prescribing exercise. Med Sci Sports Exerc. 2011;43(7):1334-599.

7. Hamar B, Coberley CR, Pope JE, Rula EY. Impact of a senior fitness program on measures of physical and emotional health and functioning. Popul Health Manag. 2013;16(6):364-72.

8. Haskell WL, Lee IM, Pate RR, Powell KE, Blair SN, Franklin BA, et al. Physical activity and public health: updated recommendation for adults from the American College of Sports Medicine and the American Heart Association. Med Sci Sports Exerc. 2007;39(8):1423-34.

9. Jordan CO, Slater M, Kottke TE. Preventing chronic disease risk factors: rationale and feasibility. Medicina (Kaunas). 2008;44(10):745-50.

10. Kraus TM, Martetschläger F, Müller D, Braun KF, Ahrens P, Siebenlist $\mathrm{S}$, et al. Return to sports activity after tibial plateau fractures: 89 cases with minimum 24-month follow-up. Am J Sports Med. 2012;40(12):2845-52.

11. Kugelman DN, Qatu AM, Haglin JM, Konda SR, Egol KA. Participation in recreational athletics after operative fixation of tibial plateau fractures: predictors and functional outcomes of those getting back in the game. Orthop J Sports Med. 2017;5(12):2325967117743916.

12. Loibl M, Bäumlein M, Massen F, Gueorguiev B, Glaab R, Perren $\mathrm{T}$, et al. Sports activity after surgical treatment of intraarticular tibial plateau fractures in skiers. Am J Sports Med. 2013;41(6):1340-7.

13. Hoekstra H, Kempenaers K, Nijs S. A revised 3-column classification approach for the surgical planning of extended lateral tibial plateau fractures. Eur J Trauma Emerg Surg. 2017;43(5):637-43.

14. de Groot IB, Favejee MM, Reijman M, Verhaar JA, Terwee CB. The Dutch version of the Knee Injury and Osteoarthritis Outcome Score: a validation study. Health Qual Life Outcomes. 2008;6:16.

15. Ornetti P, Parratte S, Gossec L, Tavernier C, Argenson JN, Roos EM, et al. Cross-cultural adaptation and validation of the French version of the Knee injury and Osteoarthritis Outcome Score (KOOS) in knee osteoarthritis patients. Osteoarthr Cartil. 2008;16(4):423-8.

16. Collins NJ, Misra D, Felson DT, Crossley KM, Roos EM. Measures of knee function: International Knee Documentation
Committee (IKDC) Subjective Knee Evaluation Form, Knee Injury and Osteoarthritis Outcome Score (KOOS), Knee Injury and Osteoarthritis Outcome Score Physical Function Short Form (KOOS-PS), Knee Outcome Survey Activities of Daily Living Scale (KOS-ADL), Lysholm Knee Scoring Scale, Oxford Knee Score (OKS), Western Ontario and McMaster Universities Osteoarthritis Index (WOMAC), Activity Rating Scale (ARS), and Tegner Activity Score (TAS). Arthritis Care Res (Hoboken). 2011;63(Suppl 11):S208-S228228.

17. Marx RG, Stump TJ, Jones EC, Wickiewicz TL, Warren RF. Development and evaluation of an activity rating scale for disorders of the knee. Am J Sports Med. 2001;29(2):213-8.

18. Tilley ST, Thomas N. What knee scoring system? J Bone Joint Surg Br. 2010. https://pdfs.semanticscholar.org/d0ed/1706e373cc b3b723873963cd96c40b508c2d.pdf. Accessed 30 Jan 2019.

19. Paradowski PT, Bergman S, Sundén-Lundius A, Lohmander LS, Roos EM. Knee complaints vary with age and gender in the adult population. Population-based reference data for the Knee injury and Osteoarthritis Outcome Score (KOOS). BMC Musculoskelet Disord. 2006;7:38.

20. Kuster MS. Exercise recommendations after total joint replacement: a review of the current literature and proposal of scientifically based guidelines. Sports Med. 2002;32(7):433-45.

21. Hall JA, Beuerlein MJ, McKee MD, Society COT. Open reduction and internal fixation compared with circular fixator application for bicondylar tibial plateau fractures. Surgical technique. J Bone Jt Surg Am. 2009;91(Suppl 2 Pt 1):74-88.

22. Ahearn N, Oppy A, Halliday R, Rowett-Harris J, Morris SA, Chesser TJ, et al. The outcome following fixation of bicondylar tibial plateau fractures. Bone Jt J. 2014;96-B(7):956-62.

23. Erdil M, Yildiz F, Kuyucu E, Sayar Ş, Polat G, Ceylan HH, et al. The effect of sagittal plane deformities after tibial plateau fractures to functions and instability of knee joint. Acta Chir Orthop Traumatol Cech. 2016;83(1):43-6.

24. Papagelopoulos PJ, Partsinevelos AA, Themistocleous GS, Mavrogenis AF, Korres DS, Soucacos PN. Complications after tibia plateau fracture surgery. Injury. 2006;37(6):475-84.

25. Rademakers MV, Kerkhoffs G, Sierevelt IN, Raaymakers ELFB, Marti R. Operative treatment of 109 tibial plateau fractures: fiveto 27-year follow-up results. J Orthop Trauma. 2007;21:5-10. 